\title{
Qualidade de vida e fatores associados em mulheres sobreviventes ao câncer do colo do útero
}

\author{
Camila Soares Lima Corrêa* \\ Maximiliano Ribeiro Guerra** \\ Anna Paula Silva Andrade*** \\ Simone Meira Carvalho**** \\ Ricardo Miranda Borges*** \\ Isabel Cristina Gonçalves Leite*****
}

\begin{abstract}
RESUMO
O tratamento para o câncer do colo do útero pode levar à ocorrência de efeitos adversos tardios, como disfunções sexuais, intestinais ou urinárias; menopausa precoce e linfedema em membro inferior, os quais podem ter impacto negativo na qualidade de vida. O objetivo do estudo foi avaliar a qualidade de vida de sobreviventes ao câncer do colo do útero, seus fatores associados e comparar a qualidade de vida com um grupo controle de mulheres sem história de câncer. O grupo câncer foi composto por mulheres com término do tratamento há três meses ( $\mathrm{n}=37$ ). O grupo controle, de base populacional, foi composto por mulheres sem história de câncer ( $\mathrm{n}=37$ ). A qualidade de vida foi avaliada pelo WHOQOL-bref e a função sexual pelo Female Sexual Function Índex. Variáveis clínicas, terapêuticas e socioeconômicas foram avaliadas por questionário desenvolvido pelos autores. Em comparação ao controle, o grupo câncer apresentou maior percentual de mulheres que viviam sem companheiro, que consideravam o relacionamento com o companheiro como ruim/regular e que apresentavam disfunções urinárias, intestinais e sexuais. Além disso, o grupo câncer apresentou piores escores nos domínios "Físico" e "Relações Sociais" do WHOQOL-bref ( $\mathrm{p}=0,03$ e 0,01, respectivamente). Foram fatores independentemente associados ao domínio "Físico": linfedema de membros inferiores e retenção urinária; e ao domínio "Relações Sociais": apoio social de amigos e estenose/encurtamento vaginal. Os resultados sugerem impacto negativo da doença e de seu tratamento sobre a qualidade de vida das sobreviventes. Deve-se investigar a qualidade de vida e os fatores que a influenciam, visando um atendimento mais integral, direcionado às necessidades das pacientes, por meio de equipe multiprofissional.
\end{abstract}

Palavras-chave: Neoplasias do colo do útero. Sobreviventes. Qualidade de vida.

\section{INTRODUÇÃO}

O câncer do colo do útero (CCU) é o quarto tipo de câncer mais comum em mulheres de todo o mundo. Em 2012, foram diagnosticados mais de500 mil novos casos da doença em todo o mundo; sendo que, $85 \%$ ocorreram em regiões menos desenvolvidas e a doença foi responsável por 7,5\% de todas as mortes por câncer feminino (FERLAY et al., 2015). No Brasil, com exceção do câncer de pele não melanoma, o CCU é o terceiro tipo de câncer mais frequente nas mulheres (BRASIL, 2015). Apesar da tendência de declínio na taxa de mortalidade por esta neoplasia nos últimos anos, ainda há grandes desigualdades no país, pois ela não ocorre em todas as regiões, como no interior do Norte e Nordeste. Além disso, continua elevada em relação a países desenvolvidos (AZEVEDO e SILVA et al., 2011; GIRIANELLI; GAMARRA; AZEVEDO; SILVA, 2014).

Devido à alta curabilidade quando detectado precocemente e aos avanços científicos e terapêuticos ao longo das últimas décadas, o número de sobreviventes a longo prazo do câncer do colo do útero está crescendo (KHALIL et al., 2015, WENZEL et al., 2005, BARKER et al., 2009). Contudo, as intervenções terapêuticas podem estar

\footnotetext{
* Programa de Pós-Graduação em Saúde Coletiva, Faculdade de Medicina, Universidade Federal de Juiz de Fora - UFJF - Juiz de Fora, MG. E-mail: camilaslcorrea@hotmail.com

** Faculdade de Medicina, Departamento de Saúde Coletiva, Universidade Federal de Juiz de Fora - UFJF, Juiz de Fora, MG.

*** Faculdade de Medicina, Universidade Federal de Juiz de Fora - UFJF, Juiz de Fora, MG.

**** Faculdade de Fisioterapia, Universidade Federal de Juiz de Fora - UFJF, Juiz de Fora, MG.

***** Faculdade de Medicina, Departamento de Saúde Coletiva. Bolsista de Produtividade em Pesquisa CNPq (processo 301101/2016-7) Universidade Federal de Juiz de Fora - UFJF, Juiz de Fora, MG.
} 
associadas à ocorrência de efeitos adversos tardios, como disfunções sexuais, intestinais ou urinárias; menopausa precoce e linfedema em membro inferior. Estes efeitos relacionados ao tratamento, por sua vez, têm impacto negativo na qualidade de vida destas mulheres (KHALIL et al., 2015; BARKER et al., 2009; LE BORGNE et al., 2013;SHUANG et al., 2014; PFAENDLER et al., 2015; LEE et al., 2016; OSAN et al., 2014).

O termo "qualidade de vida" (QV) é definido pelo The WHOQOL Group (1995) como "a percepção do indivíduo de sua posição na vida no contexto da cultura e sistema de valores nos quais ele vive e em relação aos seus objetivos, expectativas, padrões e preocupações" (p. 1405). Dentro deste contexto, a melhora da qualidade de vida passou a constituir um dos pilares do tratamento oncológico. De acordo com Fleck et al (1999), não basta acrescentar "anos à vida", é necessário acrescentar "vida aos anos". Alguns autores, inclusive, afirmam que a QV influencia na sobrevida das sobreviventes ao CCU, e, desta forma, é vista também como um fator prognóstico (ASHINGGIWA; LIM; TANG, 2010).

Nesta perspectiva, há um interesse crescente em avaliar a QV de pacientes com câncer, almejando a integralidade do cuidado. Uma vez que a QV pode ser influenciada por diversos fatores, incluindo efeitos adversos do tratamento, torna-se oportuno investigá-los, em mulheres sobreviventes ao CCU. Portanto, o objetivo do presente estudo foi avaliar a QV de mulheres sobreviventes ao CCU, seus fatores associados e, ainda, comparar a QV com um grupo controle de mulheres sem história de câncer.

\section{Material e Métodos}

Trata-se de um estudo observacional transversal com amostra composta por um grupo câncer e um grupo controle. O grupo câncer (Gcâncer) foi composto por mulheres tratadas para o CCU no período de 2005 a 2013, com conclusão do tratamento há, pelo menos, três meses na data de coleta dos dados. O recrutamento deste grupo foi efetuado em duas instituições públicas de referência para o tratamento oncológico na cidade de Juiz de Fora, Minas Gerais, Brasil. Foram incluídas as pacientes que compareceram aos serviços, cumpriram os critérios de elegibilidade e aceitaram participar da pesquisa. Para cada sobrevivente ao câncer, foi selecionada aleatoriamente, para compor o grupo controle (Gcontrole) de base populacional, uma mulher sem história de câncer, com características socioeconômicas e demográficas semelhantes. O tamanho amostral atendeu ao referencial da literatura que considera qualidade de vida superior em mulheres sem câncer ginecológico comparado com aquelas com história da doença. Assim, admitindo-se uma proporção de satisfação entre casos de $45 \%$ e nos controles de $80 \%$, com número de controles por caso de 1 , poder de teste de $80 \%$ e nível de significância de $5 \%$, estimou-se 36 casos e seus respectivos controles (SCHLESSELMAN, 1982; FRUMOVITZ et al., 2005).

Os critérios de exclusão foram: diagnóstico de transtornos psiquiátricos; doenças mentais ou neurológicas, com algum déficit cognitivo; história de outro câncer ou recidiva da doença (para as mulheres do grupo câncer) ou de morbidade importante como insuficiência renal crônica, insuficiência cardíaca, doença pulmonar obstrutiva crônica ou distúrbios osteomioarticulares graves e incapacitantes.

No grupo câncer, houve duas recusas para participação e três mulheres não atenderam aos critérios de elegibilidade devido aos seguintes motivos: estar em tratamento de recidiva, internação por doença mental e sequelas motoras por doença neurológica.

A coleta de dados foi realizada de janeiro a outubro de 2013, após aprovação pelo Comitê de Ética em Pesquisa da Universidade Federal de Juiz de Fora, sob o parecer $n^{\circ} 131.805 / 2012$.

As mulheres que participaram da pesquisa foram devidamente informadas quanto aos procedimentos, justificativa e relevância de tal estudo. As entrevistas foram realizadas por dois pesquisadores, os quais foram previamente treinados. Todas as participantes assinaram o termo de consentimento livre e esclarecido e, em seguida, os dados foram coletados através de entrevista face a face com as participantes, em espaço adequado, com a utilização dos seguintes instrumentos:

- Questionário de caracterização da amostra: desenvolvido pelos autores do presente estudo e composto por questões relacionadas a características socioeconômicas e demográficas; hábitos de vida; suporte/rede social; menopausa, sexualidade, comorbidades e sintomas autorreferidos (que no grupo câncer poderiam estar associados à doença ou ao tratamento). Para o grupo câncer, foram investigados também aspectos clínicos referentes à doença, ao tratamento realizado e ao diagnóstico de outras comorbidades, cujos dados foram obtidos por meio de busca nos prontuários.

- Questionário de avaliação da função sexual: foi utilizado o Female Sexual Function Índex para avaliar a função sexual das participantes 
sexualmente ativas - FSFI. Este questionário foi traduzido para o português e adaptado culturalmente, sendo considerado válido para avaliação da resposta sexual das mulheres brasileiras (THIEL et al., 2008) e, também, para avaliar e monitorar a função sexual de mulheres sexualmente ativas sobreviventes ao câncer, inclusive ao câncer de colo uterino (BASER et al., 2012). É composto por 19 questões e seu escore total pode variar de 2 a 36 pontos, sendo que valores mais elevados representam melhor função sexual. Para classificar a presença de disfunção sexual, utilizou-se o ponto de corte de 26 pontos (escore total $\leq 26=$ disfunção sexual) (WIEGEL et al., 2005).

- Questionário de avaliação da qualidade de vida: foi utilizado o instrumento desenvolvido pela Organização Mundial da Saúde em sua versão abreviada, o WHOQOL-bref (World Health Organization Quality of Life Instrument abreviado). Este é traduzido e validado para uso na população brasileira e se propõe a avaliar a QV de forma multidimensional e subjetiva (FLECK et al., 2000). É composto por 26 questões: duas sobre qualidade de vida global, englobando a percepção da QV e a satisfação com a saúde, e as demais representando cada uma das 24 facetas que compõem o WHOQOL-100; estas são organizadas em quatro domínios: Físico, Psicológico, Relações Sociais e Meio Ambiente (FLECK et al., 2000; KHALIL et al., 2015). Os resultados do WHOQOL-bref são analisados por domínio, pois não existe um escore total para esse questionário e os escores são padronizados para uma variação de 0 a 100 pontos (sendo 100 a melhor QV possível).

A variável dependente do estudo foi a qualidade de vida e as variáveis independentes foram características clínicas, terapêuticas, sociodemográficas, econômicas, rede social de apoio, hábitos de vida, comorbidades, status menopausal, função sexual, alterações/ disfunções autorreferidas relacionadas a funções urinárias, intestinais e linfedema de membros inferiores.

Para análise dos dados foi utilizado o software Statistical Package for Social Sciences (SPSS), versão 21.0. Por meio do teste de Kolmogorov-Smirnov, verificou-se que algumas variáveis não satisfaziam ao critério de normalidade. Desta forma, testes não-paramétricos foram utilizados para verificar a significância das associações. A fim de comparar os grupos estudados em relação a variáveis numéricas, utilizou-se o Teste de Mann Whitney e para as variáveis categóricas, foi aplicado o teste Qui-quadrado $\left(\chi^{2}\right)$ ou Exato de Fischer, quando adequado. Foi realizada regressão linear para avaliar as variáveis associadas à qualidade de vida, sendo incluídas as variáveis explicativas que apresentaram $p<0,10$ na comparação entre os grupos. Aquelas que apresentaram $\mathrm{p}<0,10$ na regressão linear simples foram, posteriormente, incluídas no modelo multivariado. Tal inclusão foi feita utilizando o método Enter. Para o modelo final multivariado, as variáveis que apresentaram $\mathrm{p} \leq 0,05$ foram consideradas estatisticamente significativas.

\section{Resultados}

Setenta e quatro mulheres participaram do estudo, divididas em dois grupos: câncer $(\mathrm{n}=37)$ e controle ( $\mathrm{n}=37$ ). A idade média das participantes foi 50,88 anos (mínimo $=24$ anos; máximo $=85$ anos, desviopadrão $= \pm 12,97$ anos) e o tempo médio de conclusão do tratamento foi 36,78 meses (variando de três a 92 meses, desvio-padrão $= \pm 25,25$ meses), sendo que a maioria $(59,5 \%)$ concluiu o tratamento há menos de três anos na data da coleta dos dados.

\section{TABEla 1}

Descrição das variáveis clínicas e terapêuticas das mulheres do grupo câncer

\begin{tabular}{|c|c|}
\hline Variáveis & $\mathrm{n}(\%)$ \\
\hline \multicolumn{2}{|l|}{ Histologia } \\
\hline Escamoso & $29(82,9)$ \\
\hline Adenocarcinoma & $6(17,1)$ \\
\hline \multicolumn{2}{|c|}{ Estadiamento (FIGO) } \\
\hline IA & $3(8,4)$ \\
\hline IB & $14(38,9)$ \\
\hline IIA & $3(8,4)$ \\
\hline IIB & $10(27,5)$ \\
\hline IIIA & $1(2,8)$ \\
\hline IIIB & $4(11,2)$ \\
\hline IVA & $1(2,8)$ \\
\hline \multicolumn{2}{|c|}{ Tratamento Local } \\
\hline Cirurgia & $6(16,2)$ \\
\hline Cirurgia e radioterapia & $17(45,9)$ \\
\hline Radioterapia exclusiva & $14(37,9)$ \\
\hline \multicolumn{2}{|c|}{ Tratamento Sistêmico (Quimioterapia) } \\
\hline Não & $19(51,4)$ \\
\hline Sim & $18(48,6)$ \\
\hline
\end{tabular}

Legenda: $\mathrm{n}$ = número absoluto; FIGO: Federação Internacional de Ginecologia e Obstetrícia.

Fonte: Os autores (2017)

Como mostra a tabela 1, a maioria das sobreviventes ao câncer teve tumor classificado histologicamente como escamoso $(82,9 \%$ - duas mulheres não tinham 
esta informação no prontuário); foi diagnosticada com estadiamento IB de acordo com a Federação Internacional de Ginecologia e Obstetrícia (FIGO) - (38,9\% - uma mulher não tinha esta informação no prontuário); submetida a tratamento cirúrgico e radioterapia adjuvante $(45,9 \%)$, sem quimioterapia $(51,4 \%)$.

Os grupos foram semelhantes em relação às variáveis socioeconômicas e demográficas, exceto para a "situação conjugal" ( $p=0,05)$, para a qual o grupo controle apresentou maior frequência de mulheres vivendo com companheiro (Gcontrole $=73 \%$; Gcâncer $=41,4 \%)$. Quanto às variáveis psicossociais, de suporte social e de hábitos de vida, $51,4 \%$ do grupo controle relatou suporte social do marido/ companheiro, enquanto no grupo câncer, 32,4\% apresentou tal apoio $(\mathrm{p}=0,09)$. O "relacionamento com o marido/companheiro" foi diferente entre os grupos $(p=0,02)$, sendo maior no grupo controle a frequência de mulheres que o consideravam como bom ou muito bom (Gcontrole $=93,1 \%$; Gcâncer $=$ $63,2 \%$ ). Além disso, o grupo câncer apresentou maior frequência de mulheres que relataram apoio social de líderes religiosos (Gcâncer: 18,9\%; Gcontrole: 2,7\%, $\mathrm{p}=0,05$ ) e de amigos (Gcâncer: 40,5\%; Gcontrole: $21,6 \%), p=0,07)$. As variáveis relacionadas à saúde (tabagismo; consumo de bebida alcoólica; diagnóstico de alguma doença crônica, como hipertensão arterial, diabetes, depressão; uso de medicamento de forma contínua) foram semelhantes entre os grupos.

Algumas variáveis, que no grupo câncer poderiam estar associadas a efeitos adversos do tratamento, foram mais frequentes neste grupo $(p<0,05)$ : menopausa; "fogachos"; estenose ou encurtamento vaginais; sangramento durante ou após a relação sexual; aumento da frequência, urgência, incontinência e retenção urinárias; diarreia, urgência e incontinência fecais; dor e muco anais; enterorragia e linfedema em membros inferiores (tabela 2). A maioria das mulheres do grupo câncer $(64,9 \%)$ apresentava menopausa relacionada ao tratamento.

Em relação à função sexual, a maioria das mulheres no grupo câncer relatou que o câncer interferiu na vida sexual $(59,5 \%)$ e que não era sexualmente ativa $(59,5 \%)$. Das mulheres sexualmente ativas, $80 \%$ apresentavam disfunção sexual no grupo câncer, enquanto este percentual foi de $7,1 \%$ no grupo controle $(p<0,01)$. Não houve diferença quanto à frequência sexual entre os grupos. Além disso, o grupo câncer apresentou menor escore total no questionário FSFI (Gcâncer: média $=21,72$, desvio-padrão $=7,40$; Gcontrole: média $=30,76$; desvio-padrão $=2,95 ; \mathrm{p}$ $<0,01)$.
TABela 2

Descrição das variáveis relacionadas à saúde, alterações/disfunções autorreferidas

\begin{tabular}{|c|c|c|c|}
\hline Variáveis & $\begin{array}{c}\text { Grupo Câncer } \\
\mathrm{n}(\%)\end{array}$ & $\begin{array}{c}\text { Grupo } \\
\text { Controle n }(\%)\end{array}$ & $\mathrm{p}$-valor \\
\hline \multicolumn{4}{|c|}{ Menopausa } \\
\hline Não & $0(0)$ & $15(40,5)$ & \multirow{2}{*}{$<0,01$} \\
\hline Sim & $37(100)$ & $22(59,5)$ & \\
\hline \multicolumn{4}{|c|}{ Menopausa relacionada ao tratamento } \\
\hline Não & $13(35,1)$ & & \\
\hline Sim & $24(64,9)$ & & \\
\hline \multicolumn{4}{|l|}{ Fogachos } \\
\hline Não & $7(18,9)$ & $22(59,5)$ & \multirow{2}{*}{$<0,01$} \\
\hline Sim & $30(81,1)$ & $15(40,5)$ & \\
\hline \multicolumn{4}{|c|}{ Vagina estreita/curta } \\
\hline Não & $13(35,1)$ & $37(100)$ & \multirow{2}{*}{$<0,01$} \\
\hline Sim & $24(64,9)$ & $0(0)$ & \\
\hline \multicolumn{4}{|c|}{ Sexualmente ativa } \\
\hline Não & $22(59,5)$ & $9(24,3)$ & \multirow{2}{*}{$<0,01$} \\
\hline Sim & $15(40,5)$ & $28(75,7)$ & \\
\hline \multicolumn{4}{|c|}{ Sangramento durante relação sexual } \\
\hline Não & $12(63,2)$ & $28(100)$ & \multirow{2}{*}{$<0,01$} \\
\hline $\operatorname{Sim}$ & $7(36,8)$ & $0(0)$ & \\
\hline \multicolumn{4}{|c|}{ Dor/ardência ao urinar } \\
\hline Não & $25(67,6)$ & $35(94,6)$ & \multirow{2}{*}{$<0,01$} \\
\hline Sim & $12(32,4)$ & $2(5,4)$ & \\
\hline \multicolumn{4}{|c|}{ Aumento frequência urinária } \\
\hline Não & $23(62,2)$ & $37(100)$ & $<001$ \\
\hline Sim & $14(37,8)$ & $0(0)$ & $<0,01$ \\
\hline Urgência & & & \\
\hline Não & $23(62,2)$ & $31(83,8)$ & 0,03 \\
\hline Sim & $14(37,8)$ & $6(16,2)$ & , \\
\hline Incontinêt & & & \\
\hline Não & $24(64,9)$ & $33(89,2)$ & 002 \\
\hline Sim & $13(35,1)$ & $4(10,8)$ & , 02 \\
\hline Incontinêt & & & \\
\hline Não & $25(67,6)$ & $22(59,5)$ & 0,14 \\
\hline Sim & $12(32,4)$ & $15(40,5)$ & 0,40 \\
\hline Retenção & & & \\
\hline Não & $29(78,4)$ & $36(97,3)$ & $00 ?$ \\
\hline Sim & $8(21,6)$ & $1(2,7)$ & 0,02 \\
\hline Hematúria & & & \\
\hline Não & $33(89,2)$ & $37(100)$ & 011 \\
\hline Sim & $4(10,8)$ & $0(0)$ & 0,11 \\
\hline Diarreia & & & \\
\hline Não & $21(56,8)$ & $37(100)$ & $<001$ \\
\hline Sim & $16(43,2)$ & $0(0)$ & $<0,01$ \\
\hline Urgência f & & & \\
\hline Não & $23(62,2)$ & $37(100)$ & \\
\hline Sim & $14(37,8)$ & $0(0)$ & $<0,01$ \\
\hline Incontinêt & & & \\
\hline Não & $29(78,4)$ & $37(100)$ & $<001$ \\
\hline Sim & $8(21,6)$ & $0(0)$ & $<0,01$ \\
\hline Constipaç & & & \\
\hline Não & $21(56,8)$ & $26(70,3)$ & 022 \\
\hline Sim & $16(43,2)$ & $11(29,7)$ & 0,22 \\
\hline Tenesmo & & & \\
\hline Não & $33(89,2)$ & $37(100)$ & 011 \\
\hline Sim & $4(10,8)$ & $0(0)$ & 0,11 \\
\hline Dor anal & & & \\
\hline Não & $29(78,4)$ & $37(100)$ & $<001$ \\
\hline Sim & $8(21,6)$ & $0(0)$ & $<0,01$ \\
\hline Muco anal & & & \\
\hline Não & $30(81,1)$ & $37(100)$ & 001 \\
\hline $\operatorname{Sim}$ & $7(18,9)$ & $0(0)$ & 0,01 \\
\hline Enterorras & & & \\
\hline Não & $28(75,7)$ & $37(100)$ & $<001$ \\
\hline Sim & $9(24,3)$ & $0(0)$ & $<0,01$ \\
\hline Linfedema & & & \\
\hline Não & $24(64,9)$ & $37(100)$ & $<001$ \\
\hline Sim & $13(35,1)$ & $0(0)$ & $<0,01$ \\
\hline
\end{tabular}




\section{TABELA 3}

Comparação entre os grupos quanto à qualidade de vida avaliada

pelo WHOQOL-bref

\begin{tabular}{lccc}
\hline WHOQOL-bref & $\begin{array}{c}\text { Grupo Câncer } \\
\text { Média } \pm \text { DP }\end{array}$ & $\begin{array}{c}\text { Grupo Controle } \\
\text { Média } \pm \text { DP }\end{array}$ & p-valor \\
\hline Percepção QV & $77,70 \pm 19,35$ & $76,35 \pm 17,62$ & 0,65 \\
Satisfação Saúde & $78,37 \pm 25,11$ & $75,67 \pm 23,19$ & 0,41 \\
Domínio Físico & $69,18 \pm 19,25$ & $78,24 \pm 17,05$ & 0,03 \\
Domínio Psicológico & $71,93 \pm 19,78$ & $74,08 \pm 17,95$ & 0,75 \\
Domínio Relações Sociais & $73,62 \pm 18,66$ & $82,64 \pm 15,01$ & 0,01 \\
Domínio Meio Ambiente & $64,42 \pm 14,25$ & $68,84 \pm 14,16$ & 0,11 \\
\hline
\end{tabular}

Legenda: DP=desvio-padrão

Fonte: Os autores (2017)

A qualidade de vida foi diferente entre os grupos nos domínios "Físico" e "Relações Sociais", sendo estes superiores no grupo controle ( $p=0,03$ e 0,01 , respectivamente) - Tabela 3. Na regressão linear simples, apresentaram associação ou tendência de associação com o domínio "Físico" as variáveis: grupo $(p=0,03)$, situação conjugal $(p=0,07)$, dor/ ardência ao urinar $(p=0,09)$, incontinência urinária ( $p$ $=0,06)$, urgência urinária $(\mathrm{p}=0,02)$, retenção urinária $(\mathrm{p}=0,006)$, urgência fecal $(\mathrm{p}=0,01)$, linfedema $(\mathrm{p}$ $=0,001)$,atividade sexual $(\mathrm{p}=0,07)$ e escore total do FSFI $(p=0,02)$. E, com o domínio "Relações Sociais": grupo $(\mathrm{p}=0,02)$, amigo como suporte social $(p=0,002)$, fogachos $(p=0,005)$, estenose/ encurtamento vaginais $(\mathrm{p}<0,001)$, dor/ardência ao urinar $(p=0,02)$, aumento da frequência urinária $(\mathrm{p}=0,003)$, urgência urinária $(0,02)$, incontinência urinária $(\mathrm{p}=0,05)$, diarreia $(\mathrm{p}=0,003)$, urgência fecal $(0,003)$, incontinência fecal $(p=0,04)$, retenção urinária $(p=0,04)$ e escore total do FSFI $(p<0,001)$. Essas variáveis foram, então, incluídas no modelo de regressão linear multivariada para cada domínio (Físico e Relações Sociais). A tabela 4 descreve as variáveis que permaneceram no modelo final.

\section{DiscusSÃo}

Os resultados do presente estudo mostraram um maior percentual de mulheres no grupo câncer que viviam sem companheiro, que consideravam o relacionamento com o companheiro como ruim/ regular e que apresentavam disfunções urinárias, intestinais e sexuais que podem estar relacionadas com o tratamento do câncer. O grupo câncer apresentou pior escore nos domínios "Físico" e "Relações Sociais" da QV. Além disso, verificou-se associação entre estes domínios de QV com alterações que poderiam representar consequências do tratamento, bem como com a rede social de apoio dos amigos, mostrando a importância destes fatores para a QV de sobreviventes do CCU.
A pior QV para o Gcâncer nos domínios "Físico" e "Relações Sociais" sugere impacto negativo do CCU e/ou seu tratamento sobre a QV das sobreviventes, o que está de acordo com outros estudos (OSANN et al., 2014; PFAENDLER et al., 2015). Neste sentido, é importante destacar que o domínio "Físico" possui um item sobre a necessidade de tratamento médico e o domínio "Relações Sociais", um item que avalia a satisfação da vida sexual. Desta forma, os resultados observados podem estar relacionados, em parte, à necessidade de acompanhamento médico especializado após o tratamento, aos efeitos adversos tardios do tratamento e à insatisfação com a vida sexual devido às disfunções sexuais secundárias à terapia.

Deve-se considerar também a possibilidade de relação entre estes resultados e a falta de orientação e suporte adequados da equipe de saúde que assiste a paciente, no que se refere à abordagem precoce dos potenciais efeitos adversos do tratamento da doença (BERNARDO et al., 2007). Além disso, destaca-se o ainda existente estigma do câncer e o fato de que a doença acomete órgão de simbologia tão importante para o sexo feminino, extremamente vinculado à atividade sexual (PENGA; RUMIN, 2008).

Variáveis relacionadas a possíveis efeitos adversos do tratamento para o grupo câncer, como estenose ou encurtamento vaginais, linfedema em membros inferiores e retenção urinária, mostraram impacto negativo na QV. O desenvolvimento de estenose ou encurtamento vaginal após o término do tratamento é, muitas vezes, considerado como o principal fator relacionado às dificuldades sexuais (WHITE, 2008). Osann et al. (2014) também verificaram que problemas ginecológicos decorrentes do tratamento para o CCU estão associados à pior QV.

Corroborando estudos anteriores, verificou-se que mulheres sobreviventes ao CCU apresentam QV significativamente inferior em comparação a mulheres do grupo controle (PARK et al., 2007; ZHOU et al., 2016; SHUANG et al., 2014). Park et al. (2007) verificaram que as sobreviventes apresentaram pobre imagem corporal, maior ansiedade em relação ao desempenho sexual, linfedema de membros inferiores e maior frequência de sintomas de menopausa em relação ao grupo controle. Segundo Bae e Park (2016), sobreviventes ao CCU tendem a apresentar disfunção sexual, o que está correlacionado à pior qualidade de vida e maiores níveis de depressão.

No presente estudo, a maioria das mulheres foi submetida ao tratamento cirúrgico e à radioterapia. De acordo com Pfaendler et al. (2015), a combinação destas modalidades aumenta o risco de sequelas a longo prazo no sistema urinário, como hipoatividade

HU Revista, Juiz de Fora, v. 43, n. 4, p. 307-3015, out./dez. 2017 


\section{TABELA 4}

Regressão linear multivariada (modelo final): variáveis que apresentaram diferença entre os grupos na análise bivariada sobre os domínios "Físico" e "Relaçõoes Sociais" do WHOQOL-bref

\begin{tabular}{lcccc}
\hline \multicolumn{1}{c}{ Variáveis independentes } & R2 & Coeficiente $\beta$ & Intervalo de confiança & p-valor \\
\hline \multicolumn{1}{c}{ Domínio Físico } & 0,29 & & & $<0,001$ \\
Linfedema em membros inferiores & & $-18,985$ & $-29,358 /-8,612$ & 0,001 \\
Retenção urinária & & $-13,046$ & $-24,836 /-1,257$ & 0,031 \\
Urgência fecal & & $-5,312$ & $-15,577 / 4,952$ & 0,306 \\
$\quad$ Domínio Relações Sociais & 0,39 & & & $<0,001$ \\
Apoio social de amigos & & 13,713 & $-6,685 / 20,740$ & $<0,001$ \\
"Fogachos" & & $-5,533$ & $-12,872 / 1,805$ & 0,137 \\
Estenose ou encurtamento vaginais & $-9,807$ & $-17,611 /-2,002$ & 0,015 \\
Aumento da frequência urinária & $-7,740$ & $-17,163 / 1,683$ & 0,106 \\
Diarreia & $-4,041$ & $-13,008 / 4,925$ & 0,372 \\
\hline
\end{tabular}

Fonte: os autores (2017)

detrusora, diminuição da sensibilidade da bexiga, capacidade vesical reduzida e incontinência urinária, que podem afetar significativamente a QV. Além disso, o linfedema de membros inferiores, outro fator que teve associação negativa com a QV no presente estudo, pode ser a sequela mais incapacitante do tratamento (PFAENDLER et al., 2015). Tal efeito adverso do tratamento leva a sensação de peso na perna, desconforto e tensão da pele e possível associação com disfunção sexual, ansiedade, depressão e diminuição da autoestima, tendo também impacto negativo na QV (TIWARI et al., 2013).Corroborando nossos resultados, Lee et al. (2016) também verificaram que o linfedema de membros inferiores foi o efeito adverso do tratamento que apresentou maior impacto na QV de sobreviventes ao CCU.

No que se refere à sexualidade, observou-se alta frequência de disfunção sexual $(80 \%)$ nas sobreviventes ao $\mathrm{CCU}$, indo ao encontro de outros estudos (BERNARDO et al., 2007; ZHOU et al., 2016; WHITE, 2008). Segundo White (2008), as dificuldades sexuais após o tratamento para o CCU apresentam uma prevalência de 50 a $80 \%$. É importante destacar que a disfunção sexual secundária ao tratamento pode levar a problemas na relação com o companheiro e, consequentemente, afetar a QV. Ratner et al. (2010) afirmam que, apesar do desejo em manter a atividade sexual, a dispareunia decorrente, principalmente, da estenose/encurtamento vaginal e da redução da lubrificação vaginal leva a paciente a sentir medo e evitar a relação sexual (RATNER et al., 2010). Quando a atividade sexual diminui, o mesmo acontece com a intimidade nos relacionamentos, devido à diminuição nos níveis de contato físico, comunicação de sentimentos e ações íntimas. Essa situação pode resultar, muitas vezes, em abandono pelos maridos, contribuindo, assim, com a carga global do câncer para a mulher e seus familiares
(BERNARDO et al., 2007; CLEARY; HEGARTY, 2011).

Como as sobreviventes ao CCU são relativamente jovens, a disfunção sexual decorrente do tratamento pode ter maior impacto na QV em relação a outros tipos de cânceres com maior prevalência em mulheres mais velhas (PFAENDLER et al., 2015). Neste contexto, alguns autores afirmam que a atividade sexual constitui um dos índices pelo qual se mede o nível de QV. Porém, ressaltam que, muitas vezes, a anamnese e o exame físico mais detalhado, enfocando aspectos sexuais, não são realizados de forma rotineira, o que pode contribuir para a persistência de dúvidas e insatisfação sexual (BERNARDO et al., 2007). Estes aspectos podem explicar, em parte, o maior percentual de disfunção sexual e o menor percentual de mulheres vivendo com companheiro e que classificam o relacionamento com o mesmo como bom/muito bom, observado no grupo câncer em relação ao grupo controle. Cabe destacar ainda que, no presente estudo, a maioria das sobreviventes ao CCU relatou que o câncer e/ou seu tratamento teve impacto na sua vida sexual.

Neste sentido, de acordo com Khalil et al. (2015), as sobreviventes ao CCU cujo status de estado civil muda após o diagnóstico de câncer de colo do útero, são mais propensas a ter QV inferiores. Os autores verificaram, em uma amostra de 110 mulheres, que $39 \%$ das mulheres casadas quando diagnosticadas estavam divorciadas no momento da entrevista, sendo que $72 \%$ atribuiu seu divórcio ao CCU. O principal motivo relatado pelas sobreviventes para não ser sexualmente ativa foi o medo de desenvolver uma recaída ou uma infecção (41\%), o que sugere a falta de orientação da equipe em relação à sexualidade (KHALIL et al., 2015).

Em contraste com os nossos resultados, Fernandes e Kimura (2010) verificaram que a situação conjugal 
foi fator preditivo para o domínio "preocupações adicionais" da QV, indicando que a presença de um companheiro gera maior conforto e suporte emocional à paciente com diagnóstico de câncer de colo uterino. Biffi e Mamede (2004) também enfatizam o parceiro sexual como uma das fontes de apoio social mais importante na assistência à saúde da mulher com câncer. Para estes autores, a relação de reciprocidade e ajuda pode favorecer recursos psicológicos e físicos, tornando a mulher mais capaz para enfrentar as dificuldades encontradas com sucesso.

No presente estudo, a referência dos amigos como fonte de apoio extrafamiliar foi fator independentemente associado à QV. Em estudo realizado por Khalil et al. (2015), apoio social e bem-estar espiritual foram os preditores de QV em mulheres sobreviventes ao CCU. Desta forma, estes resultados reforçam a importância deste tipo de apoio para a mulher. Nesta perspectiva, tal questão deve fazer parte de uma avaliação holística da paciente e, caso esta não tenha este tipo de apoio, outras fontes, como, por exemplo, apoio psicológico e espiritual, devem ser estimuladas a fim de oferecer suporte adequado. Pois, quanto mais suporte social um indivíduo obtém, melhor será o enfrentamento de situações de estresse (BIFFI; MAMEDE, 2004).

Os resultados deste estudo indicam a importância de uma investigação completa envolvendo os efeitos adversos do tratamento e a rede de suporte social da paciente em um contexto no qual a QV deve ser cada vez mais valorizada, em detrimento do tempo de vida em condição limitada ou incapacitada (FLECK et al., 2000). Neste sentido, destaca-se a necessidade de assistência multiprofissional no acompanhamento destas pacientes após o diagnóstico do câncer com o intuito de proporcionar atendimento integral voltado a todas estas necessidades que, na maioria das vezes, são negligenciadas.

Como principal limitação desta pesquisa, destacase a dificuldade no recrutamento das sobreviventes ao câncer, devido, principalmente, à desatualização dos prontuários, abandono de seguimento e ao fato de que muitas moravam em cidades vizinhas. Além disso, como não houve avaliação prévia, não se pode afirmar, com certeza, que as alterações observadas no grupo câncer são decorrentes exclusivamente do tratamento. Apesar do pequeno tamanho da amostra, pode-se demonstrar com significância estatística a hipótese de que mulheres submetidas a tratamento para o CCU apresentam algum impacto negativo na qualidade de vida, devendo, portanto, os fatores associados serem foco de intervenções.

\section{Conclusão}

Diante dos achados, percebe-se que o objetivo principal das consultas de seguimento após o tratamento para o CCU não deve ser apenas avaliar a cura ou monitorar a recidiva tumoral. Deve-se também investigar a qualidade de vida, assim como os fatores que a influencia, antes mesmo da intervenção terapêutica, com vistas a facilitar a adoção, na prática clínica, de estratégias que possam possibilitar um atendimento mais integral, direcionado às necessidades das pacientes. Logo, a equipe multiprofissional faz-se necessária no tratamento das pacientes com CCU.

Corroborando achados anteriores, os resultados obtidos neste estudo trazem informações relevantes sobre o impacto do tratamento do CCU na vida das mulheres. A partir disto, pode-se contribuir para dar maior visibilidade a estes aspectos na prática clínica, buscando guiar as intervenções e contribuir para uma melhor qualidade de vida. 


\title{
Quality of life and associated factors in women's cervical cancer survivors
}

\begin{abstract}
The treatment for cervical cancer can lead to late adverse effects such as sexual, bowel or urinary dysfunction; early menopause and lymphedema in the lower limb, which may have a negative impact on quality of life. The objective was to assess the quality of life of cervical cancer survivors, their associated factors, and to compare quality of life with a control group of women with no history of cancer. Women undergoing treatment for cervical cancer from a minimum of three months were included in the cancer group ( $\mathrm{n}=37)$. In the control group, which is population-based, were included women without history of cancer ( $\mathrm{n}=37$ ). The quality of life was evaluated using the WHOQOL-bref and the sexual function by the Female Sexual Function Index. Clinical, therapeutic and socioeconomic variables were evaluated by a questionnaire developed by the authors. In comparison to control group, the cancer group presented higher proportion of women who lived without a partner and who considered the relationship with their partner as poor / regular. In addition, cervical cancer survivors have urinary, intestinal and sexual dysfunctions. Besides, the cancer group exhibited poor score in the "Physical" and "Social Relations" domains of WHOQOL-bref ( $\mathrm{p}=0.03$ and 0.01, respectively). The factors independently associated with "Physical" domain were lower limb lymphedema and urinary retention, and with "Social Relations" domain were social support of friends and vaginal stenosis / shortening. The results suggest negative impact of the disease and its treatment on quality of life of cervical cancer survivors. Thus, it should be investigated the quality of life and the factors associated with it in order to improve patient's care, which should be performed by multiprofessional team.
\end{abstract}

Keywords: Uterine Cervical Neoplasms. Survivors. Quality of Life

\section{REFERÊNCIAS}

ASHING-GIWA, K. T.; LIM, J. W.; TANG, J. Surviving cervical cancer: Does health-related quality of life influence survival? Gynecologic Oncology, v. 118, n. 1, p. 35-42, jul. 2010.

AZEVEDO e SILVA G, et al. Tendência da mortalidade por câncer nas capitais e interior do Brasil entre 1980 e 2006. Revista de Saúde Pública, v. 45, n. 6, p. 1009-18, dez. 2011.

BAE, H.; PARK, H. Sexual function, depression, and quality of life in patients with cervical cancer. Supportive care in cancer: official journal of the Multinational Association of Supportive Care in Cancer, v.24, n.3, p.1277-1283, mar. 2016.

BARKER, C. L. et al. The impact of radiotherapy late effects on quality of life in gynaecological cancer patients. British Journal of Cancer, v. 100, n. 10, p. 1558-1565, may. 2009.

BASER, R. E.; CARTER, J.; LI, Y. Psychometric validation of the Female Sexual Function Index (FSFI) in cancer survivors. Cancer, v. 118, n. 18, p. 4606-4618, sep. 2012.

BERNARDO, B. C. et al. Disfunção sexual em pacientes com câncer do colo uterino avançado submetidas à radioterapia exclusiva. Revista Brasileira de Ginecologia e Obstetrícia, v. 29, n. 2, p. 85-90, fev. 2007.

BIFFI, R. G.; MAMEDE, M. V. Suporte social na reabilitação da mulher mastectomizada: o papel do parceiro sexual. Revista da Escola de Enfermagem da USP, v. 38, n. 3, p. 262-269, set. 2004.

BRASIL. Ministério da saúde. Instituto Nacional de Câncer José Alencar Gomes da Silva. Coordenação de Prevenção e Vigilância.
Estimativa 2016: incidência de câncer no Brasil [Internet]. Rio de Janeiro: INCA; 2015 [citado em 2017 dez 7]. Disponível em: http://www.inca.gov.br/estimativa/2016/estimativa-2016-v11. pdf. Acesso em 07 dez. 2017.

CLEARY, V.; HEGARTY, J. Understanding sexuality in women with gynaecological cancer. European journal of oncology nursing: the official journal of European Oncology Nursing Society, v. 15, n. 1, p. 38-45, feb. 2011.

FLECK, M. P. A. O instrumento de avaliação de qualidade de vida da Organização Mundial da Saúde (WHOQOL-100): características e perspectivas. Ciência \& Saúde Coletiva, v. 5, n. 1, p. 33-38, 2000.

FLECK, M. P. A. et al. Desenvolvimento da versão em português do instrumento de avaliação de qualidade de vida da OMS (WHOQOL-100). Revista Brasileira de Psiquiatria, v. 21, n. 1, p. 19-28, mar. 1999.

FERLAY, J. et al. Cancer incidence and mortality worldwide: sources, methods and major patterns in GLOBOCAN 2012. International Journal of Cancer, v. 136, n. 5, mar. 2015.

FERNANDES, W. C.; KIMURA, M. Qualidade de vida relacionada à saúde de mulheres com câncer de colo uterino. Revista Latino-americana de Enfermagem, v. 18, n. 3, p. 360367, may./jun. 2010.

FRUMOVITZ, M. et al. Quality of life and sexual functioning in cervical cancer survivors. Journal of Clinical Oncology: official journal of the American Society of Clinical Oncology, v. 23, n. 30, p. 7428-7436, oct. 2005. 
GIRIANELLI, V. R., GAMARRA, C., AZEVEDO E SILVA, G. Os grandes contrastes na mortalidade por câncer do colo uterino e de mama no Brasil. Revista de Saúde Pública, v. 48, n. 3, p. 459-67, jun. 2014.

KHALIL, J. et al. Impact of cervical cancer on quality of life: beyond the short term (Results from a single institution). Gynecologic Oncology Research and Practice, v. 2, n. 7, p. 192-197, sep. 2015.

LE BORGNE, G. et al. Quality of life in long-term cervical cancer survivors: a population-based study. Gynecologic Oncology, v. 129, n. 1, p. 222-8, apr. 2013.

LEE, Y. et al. Comparison of Quality of Life and Sexuality between Cervical Cancer Survivors and Healthy Women. Cancer research and treatment: official journal of Korean Cancer Association, v. 48, n. 4, p. 1321-1329, oct. 2016.

PARK, S. Y. et al. Quality of Life and Sexual Problems in DiseaseFree Survivors of Cervical Cancer Compared with the General Population. Cancer, v. 110, n. 12, p. 2716-2725, dec. 2007.

PENGA, V. M.; RUMIN, C. R. Vivências afetivas e o sofrimento de mulheres histerectomizadas. Omnia Saúde, v. 5, n. 2, p. 1-14, jul. 2008.

PFAENDLER, K. S. et al. Cervical cancer survivorship: longterm quality of life and social support. Clinical therapeutics, v. 37, n. 1, p. 39-48, jan. 2015.

OSANN, K. et al. Factors associated with poor quality of life among cervical cancer survivors: implications for clinical care and clinical trials. Gynecology Oncology, v. 135, n. 2, p. 266-72, nov. 2014.

RATNER, E. S. et al. Sexuality and intimacy after gynecological cancer. Maturitas, v. 66, n. 1, p. 23-26, may. 2010.
SCHLESSELMAN, J. J. Case-control studies: design, conduct, analysis. New York: Oxford University Press. 1982.

SHUANG, YE et al. A systematic review of quality of life and sexual function of patients with cervical cancer after treatment. International Journal Gynecology Cancer, v. 24, p. 1146-57, sep. 2014.

THE WHOQOL GROUP. The World Health Organization quality of life assessment (WHOQOL): position paper from the World Health Organization. Social Science \& Medicine, v. 41, n. 10, p. 1403-1409, nov. 1995.

THIEL, R. R. C. et al. Tradução para português, adaptação cultural e validação do Female Sexual Function Index. Revista Brasileira de Ginecologia e Obstetrícia, v. 30, n. 10, p. 504-10, out. 2008.

TIWARI, P. et al. Breast and gynecologic cancer-related extremity lymphedema: a review of diagnostic modalities and management options. World Journal of Surgical Oncology, v. 11, p. 237-50, sep. 2013.

WENZEL, L. et al. Quality of life in long-term cervical cancer survivors. Gynecologic Oncology, v. 97, n. 2, p. 310-17, may. 2005 .

WHITE, I. D. The Assessment and Management of Sexual Difficulties after Treatment of Cervical and Endometrial Malignancies. Clinical oncology (Royal College of Radiologists (Great Britain), v. 20, n. 6, p. 488-496, aug. 2008.

WIEGEL, M.; MESTON, C; ROSEN, R. The Female Sexual Function Index (FSFI): cross-validation and development of clinical cutoff scores. Journal of Sex $\boldsymbol{\&}$ Marital Therapy, v. 31, n. 1, p. 1-20, jan./feb. 2005.

ZHOU, W. et al. Survey of cervical cancer survivors regarding quality of life and sexual function. Journal of Cancer Research and Therapeutics, v. 12, n. 2, p. 938-944, apr./jun. 2016. 